\title{
MicroRNA-610 suppresses the proliferation of human glioblastoma cells by repressing CCND2 and AKT3
}

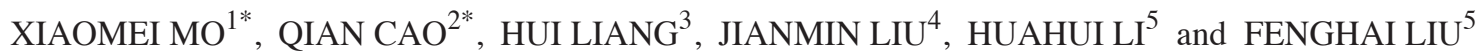 \\ ${ }^{1}$ Department of Pharmacy; ${ }^{2}$ Heart Center; ${ }^{3}$ Department of Hematology, \\ Qingdao Women and Children Hospital, Qingdao, Shandong 266034; ${ }^{4}$ Department of Neurosurgery, \\ The First Affiliated Hospital of Guangzhou University of Traditional Chinese Medicine, Guangzhou, Guangdong 510405; \\ ${ }^{5}$ Department of Laboratory Medicine of Qingdao Municipal Hospital, \\ Qingdao, Shandong 266011, P.R. China
}

Received November 30, 2014; Accepted October 14, 2015

DOI: $10.3892 / \mathrm{mmr} .2016 .4760$

\begin{abstract}
Previous studies have shown that microRNA (miR)-610 is crucial in a variety of biological processes in various types of human cancer cells. However, the role of this microRNA in glioblastoma (GBM) is presently unclear. In this study, the role of miR-610 in cell proliferation was investigated in GBM. It was demonstrated that miR-610 expression is markedly downregulated in GBM cells and GBM tissues compared with normal human astrocytes (NHAs) and normal brain tissue, respectively. Ectopic expression of miR-610 reduced the proliferation and anchorage-independent growth of GBM cells, whereas inhibition of miR-610 promoted this effect. Bioinformatics analysis further revealed cyclin D2 (CCND2) and AKT3, putative tumor promoters, as potential targets of miR-610. Data from reporter assays showed that miR-610 directly binds to the 3'-untranslated region of CCND2 and AKT3 mRNA, and represses their expression at the transcriptional and translational levels. In conclusion, the data provide compelling evidence that miR-610 functions as an anti-onco-miRNA, which is important in inhibiting cell proliferation in GBM, and its anti-oncogenic effects are mediated chiefly through direct suppression of CCND2 and AKT3 expression.
\end{abstract}

Correspondence to: Dr Huahui Li or Dr Fenghai Liu, Department of Laboratory Medicine of Qingdao Municipal Hospital, 5 Dong Hai Road, Qingdao, Shandong 266011, P.R. China

E-mail: hhliqingdao@163.com

E-mail: fenghaiqingdao@163.com

${ }^{*}$ Contributed equally

Key words: microRNA-610, human glioblastoma, cyclin D2, AKT3, cell proliferation

\section{Introduction}

Glioblastoma (GBM) is the most common and lethal type of primary brain tumor in adults (1). Despite advances in current multi-modal treatment options, the overall prognosis of patients with GBM remains poor (2); thus, there is an urgent requirement to elucidate the underlying molecular mechanisms of GBM and identify novel molecular targets for the treatment of this disease.

Recently, microRNAs (miRNAs) were found to be endogenous non-coding small RNAs, which contain 21-25 nucleotides and are involved in the post-transcriptional regulation of gene expression (3-5). An increasing number of studies have demonstrated that miRNAs are involved in the development of numerous types of human cancer, functioning as oncogenes and tumor suppressors. A number of oncogenic and tumor-suppressor proteins have been identified that are associated with the development of GBM. Akt3 is upregulated in multiple types of cancer and has been shown to be involved in the regulation of the $\mathrm{G}_{2} / \mathrm{M}$ transition (6). Cyclin D2 (CCND2) serves as a powerful oncogene that is critical in cell cycle progression and the tumorigenicity of cancer cells (7). Akt3 and CCND2 were of particular interest as their expression has been observed to be upregulated and associated with cell proliferation and cell cycle progression in several types of cancer including GBM $(7,8)$. However, the mechanism underlying this effect requires further investigation. This study explores the correlation of miR-610 with Akt 3 and CCND2 in GBM cells, and investigates its function in cell proliferation.

\section{Materials and methods}

Clinical specimens. Eight human GBM tissues and adjacent normal brain tissues (ANT) were obtained from eight patients with GBM (four men and four women; age, 40-75 years-old) at Qingdao Municipal Hospital (Qingdao, China). The study was approved by the ethics committee of Qingdao Municipal Hospital. Written informed consent was obtained from all patients. Tissue samples were collected during surgery, immediately frozen in liquid nitrogen and stored until total RNA or protein was extracted. 
Cell culture. T98G, U87, A-172,LN-229 and LN18 human GBM cell lines were purchased from the American Type Culture Collection (Manassas, VA, USA) and grown in Dulbecco's modified Eagle's medium (DMEM, Gibco-BRL, Carlsbad, CA, USA) supplemented with $10 \%$ fetal bovine serum (FBS, Sigma-Aldrich, St. Louis, MO, USA) and $100 \mathrm{U} / \mathrm{ml}$ penicillin-streptomycin (Invitrogen Life Technologies, Carlsbad, CA, USA). Normal human astrocytes (NHAs) were obtained from Lonza (Basel, Switzerland) and cultured in the provided astrocyte growth media (Lonza) supplemented with recombinant human epidermal growth factor (Invitrogen Life Technologies), insulin (Invitrogen Life Technologies), ascorbic acid (Invitrogen Life Technologies), GA-1000 (Invitrogen Life Technologies), L-glutamine (Invitrogen Life Technologies) and $5 \%$ FBS. Cell lines were cultured in a humidified incubator at $37^{\circ} \mathrm{C}$ in an atmosphere of $5 \% \mathrm{CO}_{2}$ and $95 \%$ air.

Plasmids, small interfering RNA and transfection. For ectopic expression of $\mathrm{CCN} 2$ and $\mathrm{AKT} 3, \mathrm{CCN} 2$ open reading frames (ORFs) and AKT3 ORFs with a 3'-untranslated region (UTR) were amplified using polymerase chain reaction (PCR) and subcloned into pEGFP-N3 (Invitrogen Life Technologies). To construct a luciferase reporter vector, the CCN2 and AKT3 3'-UTR fragment containing putative binding sites for miR-610 were amplified using PCR and cloned downstream of the luciferase gene in the pGL3-luciferase reporter plasmid (Promega Corporation, Madison, WI, USA). The miR-610 mimics, negative control, and miR-610 inhibitor were purchased from Genecopoeia (Rockville, MD, USA). The transfections were performed with Lipofectamine 2000 reagent (Invitrogen Life Technologies).

$R N A$ extraction and reverse transcription-quantitative PCR. Total RNA was isolated from GBM tissues, adjacent normal brain tissues, GBM cell lines and NHA cells using TRIzol reagent (Sigma-Aldrich) according to the manufacturer's instructions. RT-qPCR-based detection of mature miR-610 was performed as described previously (9). U6 small RNA was used as an internal control for normalization and quantification of miR-610 expression. The relative miR-610 expression levels after normalization to U6 small nuclear RNA were calculated

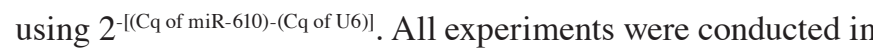
triplicate.

MTT assays and colony formation. A cell viability assay was performed to evaluate the proliferation ability of the transfected LN18 and T98G cell lines, respectively. Cells were seeded in 96-well plates in medium containing $10 \%$ FBS at $~ 4,000$ cells/well. For quantification of cell viability, cultures were stained after 1, 2, 3, 4, 5 and 6 days in MTT assays. In brief, $20 \mu \mathrm{l}$ of $5 \mathrm{mg} / \mathrm{ml}$ MTT solution (Sigma-Aldrich) was added to each well and incubated at $37^{\circ} \mathrm{C}$ for $4 \mathrm{~h}$. After the incubation period, the medium was removed from each well and the resulting MTT formazan was solubilized in $150 \mu$ l dimethyl sulfoxide (Sigma-Aldrich). The absorbance at $490 \mathrm{~nm}$ was measured in a Thermo Scientific Multiskan (Thermo Fisher Scientific Inc., Waltham, MA, USA).

For the colony formation assay, LN18 and T98G cells were plated into three $6-\mathrm{cm}$ cell culture dishes $\left(1 \times 10^{3}\right.$ cells/well $)$ and incubated for 12 days in medium containing 10\% FBS.
The colonies were washed with phosphate-buffered saline (PBS) and stained with $1.0 \%$ crystal violet (Sigma-Aldrich) for $30 \mathrm{sec}$ after fixation with $10 \%$ formaldehyde for $10 \mathrm{~min}$. The number of colonies, defined as $>50$ cells, was counted. Three independent experiments were performed and the data was calculated using a paired t-test.

Cell cycle assay. Cells were harvested, washed twice with cold PBS, fixed in ice-cold 70\% ethanol, and incubated with propidium iodide (Sigma-Aldrich) and RNase A (Sigma-Aldrich), then analyzed by fluorescence-activated cell sorting using a FACScan flow cytometer (BD Biosciences, Franklin Lakes, NJ, USA). Each sample was run in triplicate.

Luciferase assays. CCND2 and AKT3 were regulated by miR-610 through direct binding to its 3'-UTR, a series of 3'UTR fragments including the full-length wild-type 3'-UTR, binding site mutants were constructed and inserted into the psiCHECK2 luciferase reporter plasmid (Invitrogen Life Technologies). Cells were seeded in triplicate in 24 -well plates $\left(5 \times 10^{4} /\right.$ well $)$ and cultured for $24 \mathrm{~h}$. The pGL3-luciferase reporter gene plasmids pGL3-AKT3-3'-UTR (wild or mut) or pGL3-CCN2-3'-UTR (wild or mut) and the relative control-luciferase plasmid were cotransfected into the KEK293T cells with the relative control pRL-TK Renilla plasmid (Promega Corportation) using Lipofectamine 2000 reagent (Invitrogen Life Technologies). Luciferase and Renilla activities were assayed $48 \mathrm{~h}$ after transfection, the cells were lysed and the fluorescence intensity was detected using the dual luciferase assay kit (Beyotime Institute of Biotechnology, Haimen, China).

Western blotting. Protein lysates were prepared using radioimmunoprecipitation assay buffer (Cell Signaling Technology, Inc., Danvers, MA, USA), and $30 \mu \mathrm{g}$ protein was subjected to 10\% SDS-PAGE (Beyotime Institute of Biotechnology). The proteins were then transferred onto polyvinylidene difluoride membranes (EMD Millipore, Billerica, MA, USA), and the membranes were blocked in Tris-buffered saline-Tween 20 (TBST; Beyotime Institute of Biotechnology) supplemented with 5\% milk (Beyotime Institute of Biotechnology) for $2 \mathrm{~h}$ at room temperature, prior to incubation with the following primary antibodies: Anti-AKT3 (cat. no. 14982), anti-CCND2 (cat. no. 3741) (1:1,000 dilution; Cell Signaling Technology, Inc.), anti-Cyclin D1 (cat. no. 2978; 1:1,000 dilution; Cell Signaling Technology, Inc.), anti-p21 (cat. no. 2947; 1:1,000 dilution; Cell Signaling Technology, Inc.), anti-phosphorylated retinoblastoma protein (p-pRb; cat. no. 8516; 1:1,000 dilution; Cell Signaling Technology, Inc.) and anti-pRb (1:1,000; cat. no. 9313; Cell Signaling Technology, Inc.). Anti- $\beta$-actin monoclonal antibody (cat. no. 4970; 1:1,000; Cell Signaling Technology, Inc.) was used as a loading control. The membranes were subsequently washed with TBST and incubated with a horseradish peroxidase-conjugated anti-rabbit secondary antibody (cat. no. A0545; Sigma-Aldrich) for $2 \mathrm{~h}$ at room temperature. Immunocomplexes were visualized using an ECL Advance Western Blotting Detection kit (GE Healthcare Bio-Sciences, Pittsburgh, PA, USA) according to the manufacturer's instructions.

Statistical analysis. All statistical analyses except for that of microarray data were performed using SPSS 19.0 (IBM, 
A

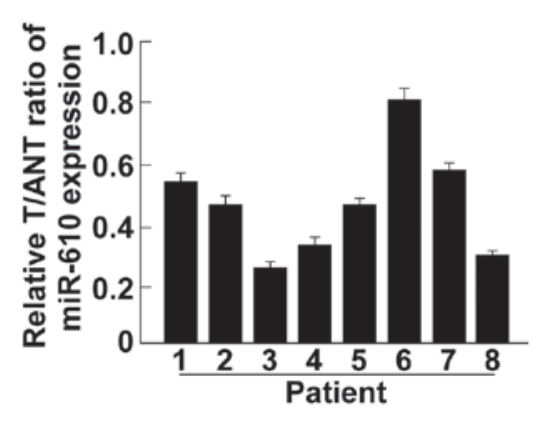

B

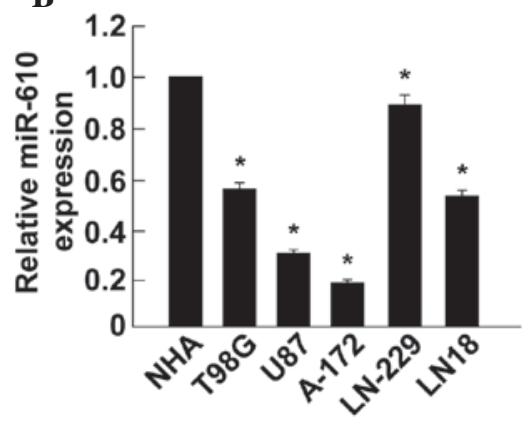

Figure 1. Expression of miR-610 in human GBM tissues and cell lines. (A) Relative miR-610 expression levels in eight paired primary GBM tissues and the matched normal brain tissues from the same patient were detected by PCR analysis. (B) RT-qPCR analysis of miR-610 expression in GBM cell lines, T98G, U87, A-172, LN-229 and LN18. Experiments were repeated at least three times. Each bar represents the mean of three independent experiments. "P<0.05 vs. NHA. miR, microRNA; GBM, glioblastoma; RT-qPCR, reverse transcription-quantitative polymerase chain reaction; NHA, normal human astrocytes; ANT, adjacent normal brain tissues.

A

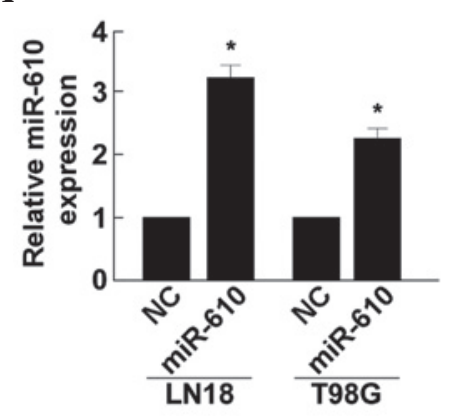

C

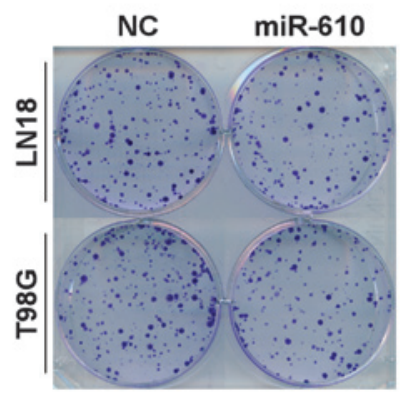

B
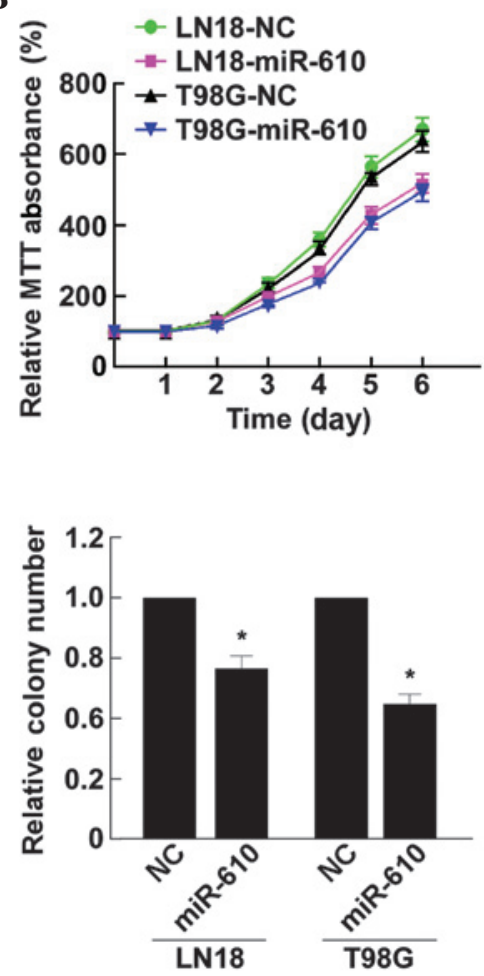

Figure 2. miR-610 upregulation inhibits GBM cell proliferation. (A) Validation of miR-610 expression levels after transfection by polymerase chain reaction analysis. (B) MTT assays revealed that upregulation of miR-610 inhibited the growth of LN18 and T98G GBM cell lines. (C) Representative micrographs (left) and quantification (right) of crystal violet-stained cell colonies. Each bar represents the mean of three independent experiments. "P<0.05 vs. NC. miR, microRNA; GBM, glioblastoma; NC, normal control.

Armonk, NY, USA). Two-tailed paired Student's t-test was used to evaluate the significance of the differences between two groups of data. $\mathrm{P}<0.05$ was considered to indicate a statistically significant difference.

\section{Results}

miR-610 expression was downregulated in GBM tissues and GBM cell lines. The expression levels of miR-610 were first evaluated in GBM tissues and GBM cells by RT-qPCR (Fig. 1). The results showed that the expression levels of miR-610 were consistently lower in the GBM tissues than in normal brain tissues, and all five tested GBM cell lines had significantly lower miR-610 levels than those in the NHAs. These results suggest that miR-610 is markedly reduced in GBM and may serve as a prognostic marker for patients with GBM.

miR-610 inhibits GBM cell proliferation and regulates the cell cycle. To further investigate the role of miR-610 in the development of GBM, the LN18 and T98G GBM cancer cell lines were transfected with miR-610 mimics, miR-610 inhibitor or the respective controls, and the effects on cellular proliferation were examined. Relative miR-610 expression was verified using RT-qPCR in miR-610-transfected cells (Fig. 2A). MTT 
A

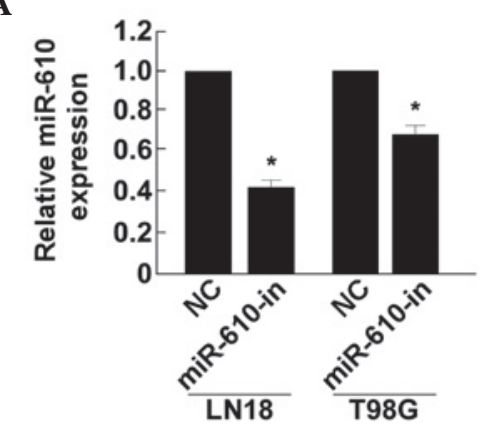

C

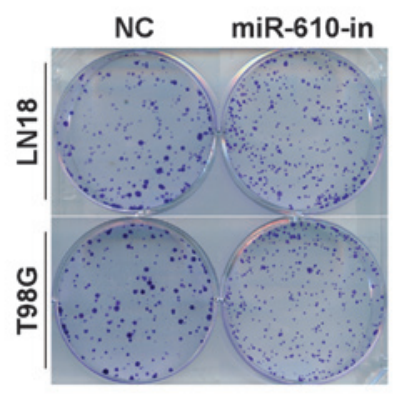

B
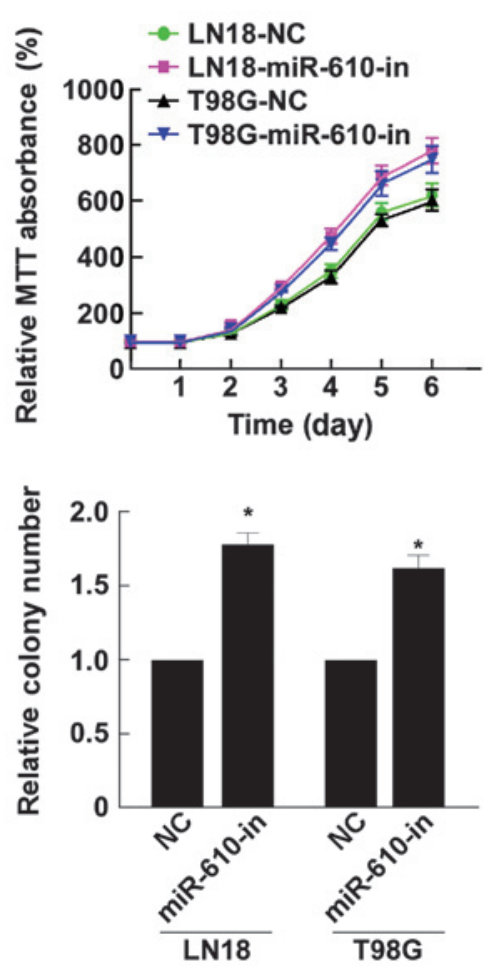

Figure 3. Inhibition of miR-610 promotes GBM cell proliferation. (A) Validation of miR-610 expression levels after transfection by polymerase chain reaction analysis. (B) MTT assays revealed that inhibition of miR-610 promoted the growth of the LN18 and T98G GBM cell lines. (C) Representative micrographs (left) and quantification (right) of crystal violet-stained cell colonies. Each bar represents the mean of three independent experiments. ${ }^{*} \mathrm{P}<0.05$ vs. NC. miR, microRNA; NC, normal control; GBM, glioblastoma; miR-610-in, miR-610-inhibitor.
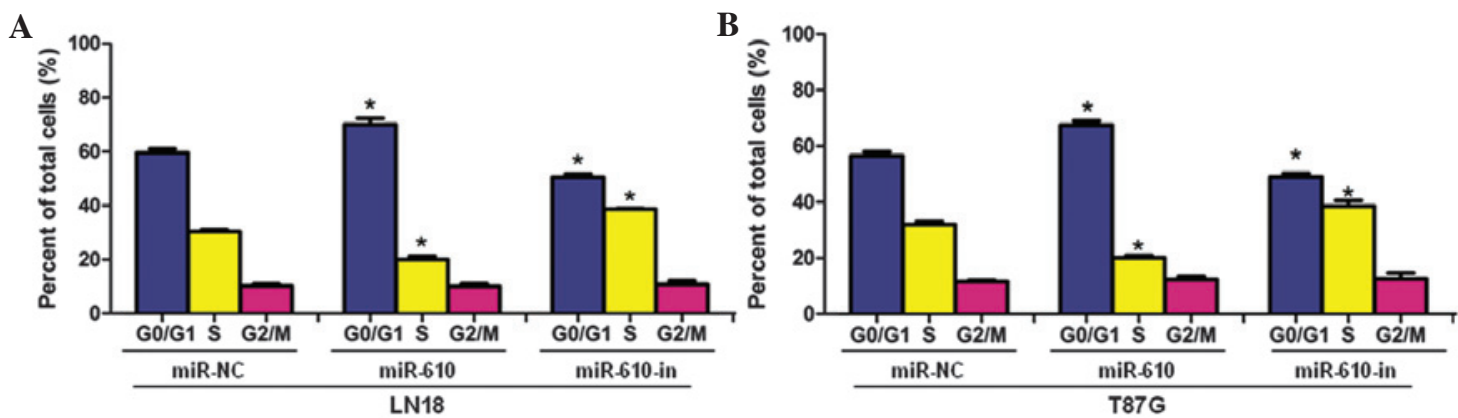

Figure 4. Cell cycle analysis was used to detect the cell cycle distribution after transfection. Cell cycle distribution in (A) LN18 cells and (B) T87G cells transfected with miR-610, miR-610-in or miR-NC. Each bar represents the mean of three independent experiments. "P<0.05 vs. NC. miR, microRNA; miR-610-in, miR-610-inhibitor; NC, normal control.

and colony formation assays revealed that overexpression of miR-610 significantly decreased the growth rate of GBM cancer cell lines, compared with the negative control-transfected cells (Fig. 2B and C). Relative miR-610 expression was verified using RT-qPCR in miR-610-in-transfected cells (Fig. 3A). By contrast, the cell growth rates and colony number of SNB19 cells transfected with miR-610 inhibitor (miR-610-in) were significantly increased compared with negative control-transfected cells (Fig. 3B and C).

To determine whether modulating cell proliferation was a result of the cell cycle, flow cytometric analysis was conducted. After $48 \mathrm{~h}$ transfection, the miR-610 mimic increased the proportion of GBM cells in the $\mathrm{G}_{0} / \mathrm{G}_{1}$-phase and decreased the proportion of GBM cells in the S-phase compared with those cells transfected with NC (Fig. 4). However, miR-610-in decreased the proportion of GBM cells in the $\mathrm{G}_{0} / \mathrm{G}_{1}$-phase and increased the proportion of GBM cells in S-phase compared with those cells transfected with NC (Fig. 4). These results showed that miR-610 reduced GBM cell tumorigenicity in vitro.

miR-610 directly targets CCND2 and AKT3 by binding to their 3'-UTR. The ability of miR-610 to impede tumor cell proliferation may be due to its ability to pleiotropically regulate genes involved in diverse aspects of proliferation. In order to identify effectors of miR-610, bioinformatic analysis was used to search for the potential regulatory targets of miR-610. CCND2 and AKT3 were two of the multiple putative target genes that were predicted by the two algorithms. CCND2 and AKT3 were of particular interest as their expression has been 
A

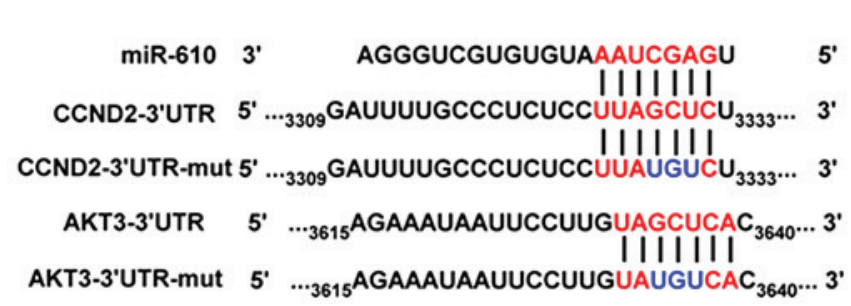

B

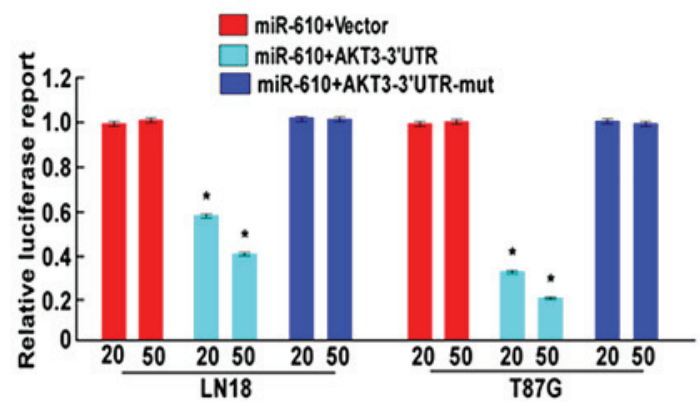

C

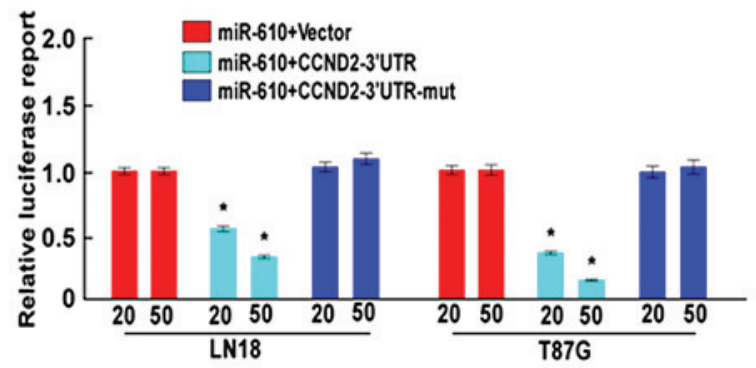

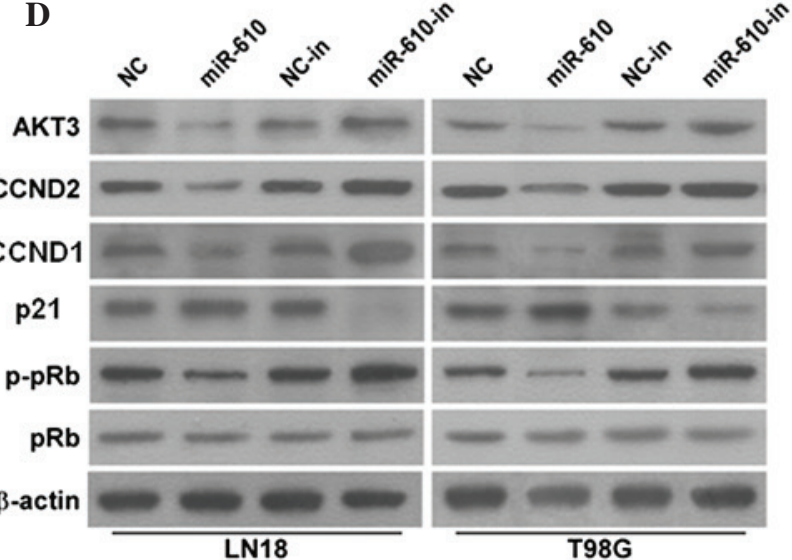

Figure 5. miR-610 suppresses CCND2 and AKT3 expression by directly targeting their 3'-UTR, which alters the levels of proteins associated with proliferation in GBM cells. (A) Predicted miR-610 target sequence in the 3'-UTR of CCND2 and AKT3 (CCND2-3'-UTR and AKT3-3'-UTR) and positions of three mutated nucleotides (blue) in the 3'-UTR of miR-610 (miR-610-mut). Luciferase reporter assay of the indicated cells co-transfected with the (B) pGL3-AKT3-3'-UTR reporter and (C) the pGL3-CCND2-3'-UTR reporter and miR-610 or miR-610-in (20 and 50 nM) oligonucleotides. (D) Western blotting analysis of expression of AKT3, CCND2, CCND1, p21, p-pRb and pRb in GBM cells. $\beta$-actin served as the loading control. "P<0.05, vs. miR-610+Vector. miR, microRNA; 1; GBM, glioblastoma; 3'-UTR, 3'-untranslated region; mut, mutant; $\mathrm{CCN}$, cyclin; $\mathrm{pRb}$, retinoblastoma protein.

observed to be upregulated and associated with proliferation in several types of cancer $(7,10)$. Using TargetScan, potential binding sites for miR-610 were located at the 3'-UTR of CCND2 and AKT3 mRNAs (Fig. 5A). In an effort to determine whether CCND2 and AKT3 were regulated by miR-610 through direct binding to its 3'-UTR, a series of 3'-UTR fragments including the full-length wild-type 3 '-UTR, binding site mutants were constructed and inserted into the psiCHECK2 luciferase reporter plasmid. The wild type and mutant vectors were co-transfected with mature miR-610 or control miRNA in HEK293T cells. miR-610 significantly decreased the luciferase activity of wild-type CCND2 and AKT3 3'-UTR but not binding site mutants CCND2 and AKT3 3'-UTR (Fig. 5B and C). This suggested that miR-610 interacts with the CCND2 and AKT3 mRNA 3'-UTR through binding to the predicted site positions.

miR-610 altered the levels of proteins associated with proliferation in GBM cells. The expression levels of a number of critical cell-proliferation regulators were also detected. As shown in Fig. 5D, the AKT3, CCND2, CCND1 and p-pRb levels in GBM cells were all significantly downregulated by miR-610, and upregulated by the miR-610 inhibitor, while the expression of p21 was upregulated in cells transfected with miR-610 and downregulated in cells transfected with the miR-610-in. Thus providing further evidence that miR-610 is important in GBM cell proliferation. The results indicated that miR-610 functionally modulates the cellular proliferation regulators, AKT3, CCND2, CCND1, p-pRb and p21.

\section{Discussion}

MicroRNAs (miRNAs) are a class of small (22 nucleotides) non-coding highly conserved single-stranded RNAs that repress protein translation through binding to the 3'-UTR of their target mRNAs in a sequence-specific manner. They are important in multiple biological processes, including the regulation of cell proliferation, cycle, differentiation, survival and apoptosis $(4,11,12)$. Over the past few decades, although certain studies have investigated the role of miR-610 in cancer cells $(13,14)$, its function has not been fully elucidated in GBM cells. In this study, it was demonstrated that expression of miR-610 was markedly downregulated in GBM tissues and cells. Furthermore, ectopic expression of miR-610 decreased the cell proliferation of GBM cells, whereas miR-610-in had the opposite effect. Thus, the data are consistent with previously published studies which associated changes in miR-610 expression with tumor formation and progression $(13,14)$, implicating miR-610 as an anti-onco-miR. It was demonstrated that miR-610 may be essential during the development of GBM.

More specifically, bioinformatic algorithms predicted that AKT3 and CCND2 were bona fide target genes of miR-610. Evidence has recently emerged to suggest that AKT3 and 
CCND2 act as tumor promoter genes, and their expression is frequently increased in a variety of human cancers, including ovarian cancer, bladder carcinoma, colorectal cancer and glioblastoma (6,15-19). Progression through each phase of the cell cycle requires the formation of a specific complex, comprising a cyclin and a cyclin-dependent kinase (CDK). Current research suggests that $\mathrm{CCND1}$ and $\mathrm{CCND} 2, \mathrm{CDK}$ regulators important for regulating $G_{1} / S$ transition, are required for the tumorigenesis of GBM $(7,20)$. The CCND1/CDK4 system is known to be critical in the progression of cells from $G_{1}$ to $\mathrm{S}$-phase by phosphorylating pRB $(21,22)$. Previous studies revealed that the AKT signal transduction cascade was required for cell-cycle progression through the $\mathrm{G}_{1}$-phase. In addition, activation of AKT decreases the cellular levels of p21, and induces CCND1 expression, thereby promoting cell proliferation (23). Data showed that the negative regulation of AKT3 and CCND2 by miR-610 leads to downregulation of CCND1 and p-pRb, and upregulation of p21. Altogether, the results indicated that miR-610 functionally modulates cell cycle regulators, CCND1, p21 and p-pRb, and thus is relevant in cell proliferation and cell cycle progression.

In conclusion, in this study, a significantly lower expression level of miR-610 was found in GBM tissues and cells compared with adjacent non-cancer tissues and NHAs. The anti-proliferative effect of miR-610 was observed, as well as its anti-oncogenic role in GBM. This may occur through post-translationally downregulating AKT3 and CCND2 expression by targeting their mRNA 3'-UTR, resulting in downregulation of CCND1 and p-pRb, and upregulation of $\mathrm{p} 21$. Thus, miR-610 may be a candidate for molecular targeted therapy and as a biological marker to predict the treatment outcome in patients with GBM.

\section{Acknowledgements}

The present study was supported by the Qingdao Key Health Discipline Development Fund.

\section{References}

1. Wen PY and Kesari S: Malignant gliomas in adults. N Engl J Med 359: 492-507, 2008.

2. Stupp R, Mason WP, van den Bent MJ, Weller M, Fisher B, Taphoorn MJ, Belanger K, Brandes AA, Marosi C, Bogdahn U, et al: Radiotherapy plus concomitant and adjuvant temozolomide for glioblastoma. N Engl J Med 352: 987-996, 2005.

3. Baek D, Villén J, Shin C, Camargo FD, Gygi SP and Bartel DP: The impact of microRNAs on protein output. Nature 455: 64-71, 2008.

4. Bartel DP: MicroRNAs: Genomics, biogenesis, mechanism and function. Cell 116: 281-297, 2004.

5. Miska EA: How microRNAs control cell division, differentiation and death. Curr Opin Genet Dev 15: 563-568, 2005.

6. Cristiano BE, Chan JC, Hannan KM, Lundie NA, Marmy-Conus NJ, Campbell IG, Phillips WA, Robbie M, Hannan RD and Pearson RB: A specific role for AKT3 in the genesis of ovarian cancer through modulation of $\mathrm{G}(2)-\mathrm{M}$ phase transition. Cancer Res 66: 11718-11725, 2006.
7. Koyama-Nasu R, Nasu-Nishimura Y, Todo T, Ino Y, Saito N, Aburatani H, Funato K, Echizen K, Sugano H, Haruta R, et al: The critical role of cyclin D2 in cell cycle progression and tumorigenicity of glioblastoma stem cells. Oncogene 32: 3840-3845, 2013.

8. Takai H, Masuda K, Sato T, Sakaguchi Y, Suzuki T, Suzuki T, Koyama-Nasu R, Nasu-Nishimura Y, Katou Y, Ogawa H, et al: 5-Hydroxymethylcytosine plays a critical role in glioblastomagenesis by recruiting the CHTOP-methylosome complex. Cell Rep 9: 48-60, 2014.

9. Li S, Chen T, Zhong Z, Wang Y, Li Y and Zhao X: microRNA-155 silencing inhibits proliferation and migration and induces apoptosis by upregulating BACH1 in renal cancer cells. Mol Med Rep 5: 949-954, 2012.

10. Chang CC, Lin BR, Wu TS, Jeng YM and Kuo ML: Input of microenvironmental regulation on colorectal cancer: Role of the CCN family. World J Gastroenterol 20: 6826-6831, 2014.

11. Formosa A, Markert EK, Lena AM, Italiano D,Finazzi-Agro' E, Levine AJ, Bernardini S, Garabadgiu AV, Melino G and Candi E: MicroRNAs, miR-154, miR-299-5p, miR-376a, miR-376c, miR-377, miR-381, miR-487b, miR-485-3p, miR-495 and miR-654-3p, mapped to the 14q32.31 locus, regulate proliferation, apoptosis, migration and invasion in metastatic prostate cancer cells. Oncogene 30: 5173-5182, 2014.

12. Lehmann U, Streichert T, Otto B, Albat C, Hasemeier B, Christgen H, Schipper E, Hille U, Kreipe HH and Länger F: Identification of differentially expressed microRNAs in human male breast cancer. BMC Cancer 10: 109, 2010.

13. Jin J, Li C, You J and Zhang B: miR-610 suppresses lung cancer cell proliferation and invasion by targeting GJA3. Zhonghua Zhong Liu Za Zhi 36: 405-411, 2014 (In Chinese).

14. Wang J, Zhang J, Wu J, Luo D, Su K, Shi W, Liu J, Tian Y and Wei L: MicroRNA-610 inhibits the migration and invasion of gastric cancer cells by suppressing the expression of vasodilator-stimulated phosphoprotein. Eur J Cancer 48: 1904-1913, 2012.

15. Beltran AL, Ordonez JL, Otero AP, Blanca A, Sevillano V, Sanchez-Carbayo M, Kirkali Z, Cheng L, Montironi R, Prieto R and De Alava E: Fluorescence in situ hybridization analysis of CCND3 gene as marker of progression in bladder carcinoma. J Biol Regul Homeost Agents 27: 559-567, 2013.

16. Soung YH, Lee JW, Nam SW, Lee JY, Yoo NJ and Lee SH: Mutational analysis of AKT1, AKT2 and AKT3 genes in common human carcinomas. Oncology 70: 285-289, 2006.

17. Agarwal E, Brattain MG and Chowdhury S: Cell survival and metastasis regulation by Akt signaling in colorectal cancer. Cell Signal 25: 1711-1719, 2013.

18. Paul-Samojedny M, Suchanek R, Borkowska P, Pudełko A, Owczarek A, Kowalczyk M, Machnik G, Fila-Daniłow A and Kowalski J: Knockdown of AKT3 (PKB $\gamma)$ and PI3KCA suppresses cell viability and proliferation and induces the apoptosis of glioblastoma multiforme T98G cells. Biomed Res Int 2014: 768181, 2014.

19. Parry PV and Engh JA: The role of cyclin-d2 in the tumorgenesis of glioblastoma. Neurosurgery 71: N22-N23, 2012.

20. Guo Y, Yan K, Fang J, Qu Q, Zhou M and Chen F: Let-7b expression determines response to chemotherapy through the regulation of cyclin D1 in glioblastoma. J Exp Clin Cancer Res 32: 41, 2013.

21. Terada Y, Inoshita S, Nakashima O, Kuwahara M, Sasaki S and Marumo F: Cyclins and the cyclin-kinase system-their potential roles in nephrology. Nephrol Dial Transplant 13: 1913-1916, 1998.

22. Féliers D, Frank MA and Riley DJ: Activation of cyclin D1-Cdk4 and Cdk4-directed phosphorylation of RB protein in diabetic mesangial hypertrophy. Diabetes 51: 3290-3299, 2002.

23. Roy SK, Srivastava RK and Shankar S: Inhibition of PI3K/AKT and MAPK/ERK pathways causes activation of FOXO transcription factor, leading to cell cycle arrest and apoptosis in pancreatic cancer. J Mol Signal 5: 10, 2010. 